\title{
Malaria vaccines 1985-2005: a full circle?
}

\author{
Geoffrey A. Targett
}

Gates Malaria Partnership, Department of Infectious and Tropical Diseases, London School of Hygiene \& Tropical Medicine, 50 Bedford Square, London, UK, WC1B 3DP

Few who were actively engaged in malaria vaccine research 20 years ago (including myself) would have imagined that, in 2005 , there would still be a prediction of a 10-20-year horizon before vaccines become part of malaria-control strategies. Why is it still proving so challenging to produce effective vaccines?

\section{Prospects}

In the debate 'Towards a malaria vaccine', which was published in 1985 in Parasitology Today, the conclusions drawn by the authors of the four articles were optimistic: (i) that the tandem-repeat sequences that constitute a substantial and immunodominant part of the circumsporozoite protein (CSP) of Plasmodium falciparum provided a promising candidate for vaccine development [1]; (ii) that several different asexual blood-stage antigens or fragments of them could form the basis of an effective vaccine [2]; (iii) that the first phase of clinical testing of a transmission-blocking vaccine could soon be considered [3]; and (iv) that the prospects for practical malaria vaccines had moved into the realm of feasibility in only ten years [4].

\section{Pre-erythrocytic-stage vaccines}

By 1985, it had already been shown experimentally and in humans that irradiated sporozoites could confer a strong (sterile) immunity [5]. The subsequent focus became the development of recombinant or peptide vaccines based on the CSP antigen and other sporozoite surface antigens that would reproduce or enhance the level of immunity achieved with the whole attenuated organism. Notably, it was shown that the C-terminal region of the CSP that flanks the naturally immunodominant tandem-repeat region of the molecule contains $\mathrm{CD}^{+}$and $\mathrm{CD}^{+}{ }^{+}$T-cell epitopes and that these were incorporated into vaccine constructs. However, most of the small-scale vaccine trials subsequently conducted obtained disappointing results [6]. Vaccine technologies have advanced considerably since then [7,8] (Box 1), and there is still a range of recombinant and peptide CSP vaccines under investigation [see the World Health Organization portfolio of candidate malaria vaccines (http://www.who.int/vaccine_ research/documents/en/malaria_table.pdf)]. As yet, only one construct (RTS,S) has progressed far in clinical trials.

Corresponding author: Targett, G.A. (geoff.targett@lshtm.ac.uk)

Available online 31 August 2005
Box 1. Examples of malaria vaccine technologies currently employed in clinical trials

Prime-boost (clinical Phase II)

Priming of the immune response uses antigens expressed as plasmid DNA or in attenuated viral vectors such as fowlpox or adenovirus. Boosting involves use of attenuated viral vectors such as modified vaccinia Ankara (MVA) expressing the same antigen(s) [9].

Virosomes (clinical Phase I)

Spherical unilamellar phospholipids representing liposomes and incorporating antigen in the membrane. The vaccine PEV3A contains peptides of the CSP and of part of AMA-1 of Plasmodium falciparum (http://www.pevion.com/downloads/pevion/doc/Pevion $\% 20$ Phasel\%20Malaria_061804_en.pdf).

Virus-like particles (clinical Phase I-II)

A recombinant virus-like particle (VLP) comprising modified hepatitis B core protein with B- and T-cell epitopes of $P$. falciparum. A single dose administered with Montanide ISA 720 adjuvant did not protect subjects [49].

\section{Long synthetic polypeptides (clinical Phase I)}

Peptides based on the $C$ terminus of $P$. falciparum CSP combined with alum or Montanide ISA 720 adjuvant-induced antibody, $\mathrm{CD}^{+}$ T-cell and $\gamma$-interferon responses [50]. Plasmodium vivax CSP peptides are similarly immunogenic. Long peptides containing B- and T-cell epitopes of MSP-3 induced monocyte-dependent parasite inhibitory Immunoglobulin G responses [9].

\section{Co-expression of recombinant polypeptides}

(clinical Phase II)

RTS, S consists of a single polypeptide corresponding to substantial parts of $P$. falciparum CSP and the hepatitis B surface antigen, expressed in yeast. An important component is the adjuvant ASO2A, which consists of an oil-in-water emulsion incorporating the immunostimulants monophosphoryl lipid $A$ and the saponin derivative QS21. (See main text for details of results of the trials.)

\section{Subunit recombinant blood-stage antigens (clinical Phase I-II)}

The C-terminal region of MSP-1 (MSP-1 ${ }_{42}$ ) given with ASO2A adjuvant was safe and immunogenic. Challenge studies in adults gave no protection [9]; Phase I and II studies in children in Kenya are in progress. A similar Phase I study with a subunit AMA-1 vaccine and a Phase I trial with an MSP-1-AMA-1 chimaera have been conducted [9].

\section{Recombinant transmission-blocking vaccine} (clinical Phase I)

The Pvs25 ookinete antigen of $P$. vivax was expressed in yeast, purified and adsorbed onto Alhydrogel ${ }^{\circledR}$. Three immunizing doses induced antibodies that blocked transmission significantly [45]. A similar approach with the $P$. falciparum homologue is being tested. 
This vaccine consists of a large segment of the tandemrepeat region of the CSP, in addition to the T-cell epitopes of the flanking region, expressed with the hepatitis B surface antigen in yeast. The vaccine is given with a three-component adjuvant [9]. The results from trials in Gambian adults [10] and Mozambican children [11] are encouraging, with a reported protection in children of $30 \%$ in the time to the first clinical episode of malaria, and $58 \%$ protection against severe malaria. However, these trials have raised questions of fundamental importance to the development of all vaccines that might partly explain the slow progress in bringing candidate vaccines successfully through clinical trials - namely, is there adequate induction of an appropriate memory T-cell response to provide the long-lived protection that is required? This seems not to be the case in the Gambian study [10], and the Mozambican results are also open to the same interpretation [12]. This must be addressed in the further series of Phase II trials of RTS,S being planned.

Trials of vaccines have (understandably) been carried out in an empirical way, with the focus in the 1980s being on antibodies as the mediators of protection. For the past 15 years, there has been increasing recognition of the need to determine at a cellular level which immune responses must be induced to achieve robust protection (Figure 1). The demonstration that protection induced by irradiated sporozoites involves both antibody and cell-mediated effector mechanisms $[5,13]$ focused attention on liver stages and the induction of both $\mathrm{CD} 4^{+}$and $\mathrm{CD} 8^{+}$T-cell responses that would be effective against multiplicative intrahepatic schizonts.

The development of a prime-boost strategy for vaccination has proved particularly effective in inducing such T-cell responses. The vaccines tested using this approach consist of malaria antigens presented as plasmid DNA or expressed by attenuated viral vectors, given in a sequence that provides strong priming and then boosting of the cellular immune responses [7]. This approach, pioneered in malaria by Hoffman and colleagues at the US Naval Medical Research Institute [14] and by Hill and colleagues [15], has involved the use of complex antigens: for example, the sporozoite surface protein TRAP (thrombospondin-related adhesion protein) fused to a multi-epitope (ME) string of $\mathrm{B}$ cell, $\mathrm{CD}^{+}$and $\mathrm{CD} 4^{+}$epitopes from sporozoite and liver-stage antigens. Immunization of malaria-naïve volunteers has given full protection in a small number of cases and, where this did not occur, it has provided evidence of a substantial reduction in the number of liver-stage parasites and, hence, the number of merozoites released to invade the blood [16]. Disappointingly, trials in an endemic area following the same regimes have not induced any protection so far, despite greatly enhanced T-cell responses as measured by $\gamma$-interferon enzyme-linked immunospot (ELISPOT) assays [17]. Again, this raises the issue of the cellular basis of the immune responses required and, perhaps, those to be avoided. Further clinical trials are in progress.

While the trials with RTS,S and other pre-erythrocytic recombinant and peptide vaccines continue with varying

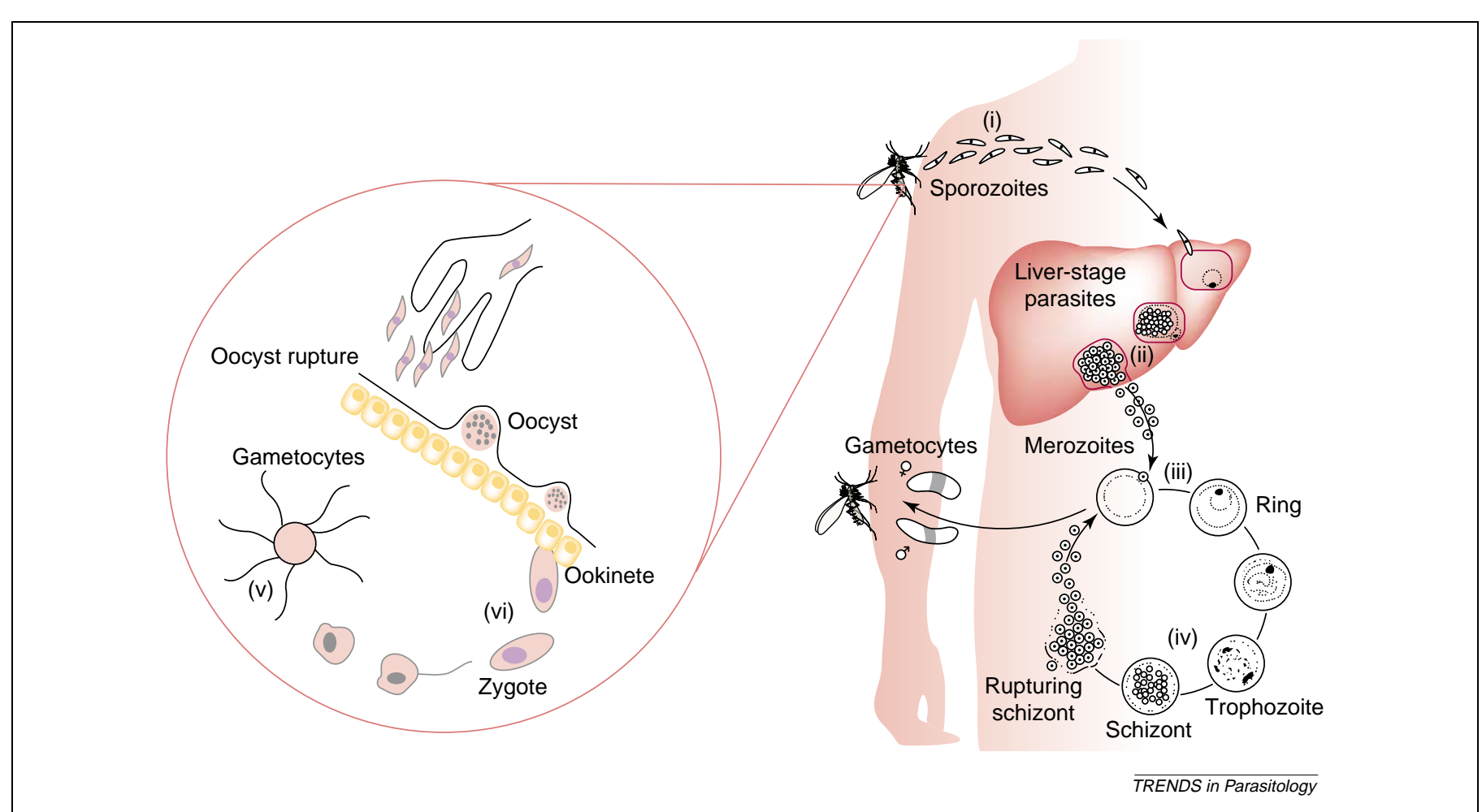

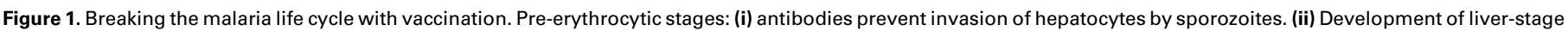

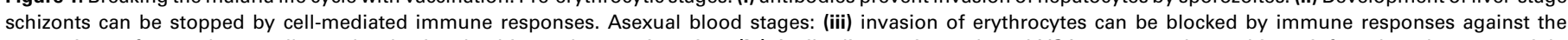

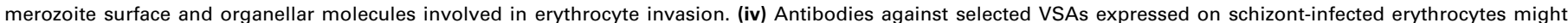
prevent (Plasmodium falciparum) parasite development, probably by preventing cytoadherence. Transmission-blocking: (v) antibodies to gamete antigens prevent

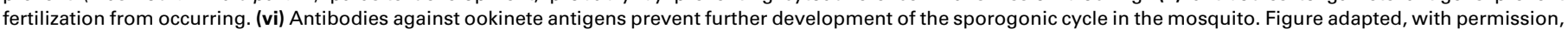
from Ref. [51]. 
schedules, antigen combinations and adjuvants, attempts are being made to exploit the findings from 30 years ago to produce a whole-organism (attenuated sporozoite) vaccine [18]. Of interest is the recent demonstration that irradiated sporozoites enhance the ability of splenic antigenpresenting cells to process and present sporozoite antigens and to prime effector T-cell responses [19]. This might provide a lead as to the type of immune response required for good protection to be achieved [20]. Mueller et al. [21] recently described a different approach for producing attenuated sporozoites. They used gene disruption to produce sporozoites deficient in the uis 3 gene, which codes for a protein thought to be involved in sporozoite motility. Immunization with the uis3-deficient sporozoites of the rodent malaria parasite Plasmodium berghei gave complete protection. The problems associated with the largescale production of both radiation- and genetically attenuated sporozoites are considerable [22] but the whole-organism approach to vaccination remains a valid alternative to the subunit approaches because it has been shown to provide protection in humans for at least ten months [5].

\section{Asexual-blood-stage vaccines}

Although improved vaccine technologies have been central to attempts to develop more-effective pre-erythrocytic-stage vaccines (Box 1), studies of asexual blood stages during the past 20 years have revealed a detailed array of molecules associated with parasite development and pathogenesis, and the natural acquisition of immunity. At the molecular level, the advances have been remarkable during this time and are set to continue with the genomic and proteomic data that are accumulating [23,24]. That said, the asexual-bloodstage target antigen that is undergoing the most intensive investigation is still, as in 1985 , the merozoite surface protein MSP-1.

It was already recognized in 1985 that the asexual blood stages are the major target of naturally acquired immune responses, that immunization with merozoite antigens involved in red blood cell invasion (Figure 1) is likely to be complicated by antigenic diversity and that the preferred molecules or epitopes for inclusion in a vaccine might, consequently, be those that are nonvariant [25]. The majority view today would not be very different and it is interesting to consider why the first asexual-blood-stage vaccines are going into clinical trials only now.

Naturally acquired immunity in a highly endemic setting is a state of premunition. Individuals remain 'immune' and asymptomatic because they have low-grade chronic infections [25]. This acquired immunity is induced predominantly by antigens that are polymorphic or that undergo clonal antigenic variation [26]. This contributes to the chronic state of infection by enabling the parasite to evade immune responses. In one of the few clinical trials carried out with asexual-blood-stage antigens, vaccination with MSP-1, MSP-2 and the ring-infected erythrocyte surface antigen (RESA) reduced parasite density significantly, but this was a strain-specific effect [27]. Apical membrane antigen (AMA)-1, another vaccine candidate that was known in 1985, is also highly polymorphic and, similarly, induces strain-specific immunity [28].
A fair conclusion that is drawn frequently from studies of natural immunity is that, for vaccine development, it would be better to focus on cryptic epitopes [24] rather than epitopes of the highly immunogenic polymorphic or clonally variant domains [29]. However, some promising liver- and blood-stage candidate vaccine molecules have been selected after analysis of naturally acquired immune responses [30,31]. Also, the variant surface antigens (VSAs) of parasites that cause severe disease are different from and more immunogenic than those isolated from cases of mild malaria [32]. The possibility of exploiting this as a vaccine strategy is considered later.

Some of the candidate antigens under investigation are poorly immunogenic because they have a limited number of T-cell determinants; hence, they are MHC restricted and induce an immune response in only subsets of the population. There are ways to overcome this that have been known for a long time, notably coupling the relatively small vaccine molecules to carriers containing T-cell epitopes [33]. Other features of the asexual blood stage of infection that could compromise the induction of a strong response to vaccines are that parasitized erythrocytes can suppress maturation of dendritic cells, thus impairing antigen presentation to $\mathrm{T}$ cells [34], and can cause apoptosis of malaria-specific T cells and B cells [25]. Infants and young children are the principal target population for malaria vaccines, and maternally derived antibodies might impair the ability of the vaccinated infant to make an adequate antibody response to some candidate vaccines. Vaccine-dosing schedules could overcome this inhibitory effect on antibody production, and $\mathrm{CD}^{+}{ }^{+}$T-cell responses do not seem to be impaired [35].

These various hurdles pose a considerable problem for subunit vaccine design and probably explain the relative lack of experimental success of blood-stage vaccines, despite the recognition in 1985 that immunization with processed fragments of what is now called MSP- 1 induces protective immune responses [2].

The emphasis on developing candidate asexual-bloodstage vaccines continues to be based on subunit strategies (Box 1) but, stimulated by studies from more than 20 years ago, malaria-naïve volunteers were recently shown to be fully protected against homologous challenge if immunized using extremely low-dose infections by inoculation of $\sim 30$ erythrocytes infected with $P$. falciparum on three occasions, with each infection being drug cured eight days after induction. Of particular interest is the observation that protection seemed to be cell mediated rather than antibody mediated [36]. This has the appearance of an innate, cytokine-mediated protection induced early in the infection [37]. However, this re-awakening of the wholeorganism approach to vaccination against blood stages requires further investigation to see whether there is a feasible way of exploiting it, perhaps by focusing on antigens that are targets of cell-mediated immunity [38].

In addition, studies of the $P$. falciparum VSAs that are located on the infected erythrocyte surface have revealed a potentially novel approach to vaccination (Figure 1). These variant antigens are responsible for sequestration of the infected red blood cells and for antigenic variation of the parasites. A unique subset of VSAs that binds to 
chondroitin sulfate A has been identified on placental isolates of $P$. falciparum. The best-characterized variant is VAR2CSA, which is sex specific and highly transcribed $[39,40]$. Also, as we have seen, the variant proteins from parasites that cause severe malaria in non-immune patients differ from those expressed by parasites that cause uncomplicated malaria $[32,41]$. Targeting these small groups of variants offers the intriguing possibility of developing vaccines specifically to protect women in pregnancy and the children most at risk of severe disease.

\section{Transmission-blocking vaccines}

It was known more than 20 years ago that immune responses to antigens expressed on macrogametes and microgametes of various Plasmodium species modulate transmission to mosquitoes [3] (Figure 1). The Pfs230 and Pfs 48/45 gamete antigens of $P$. falciparum have been studied in detail, and antibodies against both block transmission extremely effectively $[42,43]$. Parallel studies revealed Pfs 25 and Pfs28 antigens, and their homologues in Plasmodium vivax and other Plasmodium species, which are expressed only on the zygote and ookinete stages within the mosquito (Figure 1). Antibodies against these antigens also block sporogonic (oocyst) development in the mosquito extremely effectively [43]. The conformational structure of both protein families has complicated their cloning and expression in an immunogenic form that is suitable for testing as a candidate vaccine. An advantage when testing this type of vaccine is that animals can be immunized with the selected vaccine construct and then their sera can be tested for transmission-blocking activity against the human parasite in a membrane-feeding assay [44]. Phase I clinical trials of recombinant forms of the ookinete antigens of $P$. vivax Pvs25 and Pvs28 - have started [45] and the homologous $P$. falciparum vaccines will soon be tested. Forthcoming trials will probably focus on series of Phase I safety and immunogenicity trials of different vaccine constructs, but always with the option of testing sera from the volunteers for efficacy in the membrane-feeding assay. Beyond that, transmission-blocking vaccines might be of greatest use in protecting the efficacy of other malaria vaccines by preventing transmission of vaccine-resistant strains of parasite. The recent demonstration [46] that the proteome of male gametocytes contains $36 \%$ male-specific proteins and that the proteome of female gametocytes contains $19 \%$ female-specific proteins offers the potential to select new targets for sexual-stage-specific vaccines.

\section{Future perspectives}

Effective vaccines for malaria must reproduce or, even better, improve naturally acquired immunity. However, the latter, which is directed primarily against asexual blood stages, requires repeated exposure and involves persistence of infection, responses to complex antigenic polymorphisms, immune modulation and immune evasion. On that basis, it has been argued that, to be effective, a vaccine should not induce a sterilizing immunity, certainly against the clinically important phase of infection [47]. The goal for pre-erythrocytic (and transmission-blocking) vaccines remains the prevention of all parasite development, but this is far from being achieved at present. It is doubtful whether any of the vaccines currently scheduled for clinical trials will, on their own, have the efficacy and long-term effectiveness to justify widescale use. This will probably be achieved only with combination, multi-component vaccines.

The first generation of malaria vaccines will probably be used to supplement strategies of vector control and drug treatment for reducing rates of morbidity and mortality [48]. A vaccine that is good enough to be an effective alternative to treatment and vector control remains a more distant goal and might require another 20 years to perfect.

\section{Acknowledgements}

I am grateful for the helpful comments made by Brian Greenwood and the referees about the manuscript.

\section{References}

1 Nussenzweig, R.S. and Nussenzweig, V. (1985) Development of a sporozoite vaccine. Parasitol. Today 1, 150-152

2 Anders, R.F. (1985) Candidate antigens for an asexual blood-stage vaccine. Parasitol. Today 1, 152-155

3 Targett, G.A.T. and Sinden, R.E. (1985) Transmission blocking vaccines. Parasitol. Today 1, 155-158

4 Wernsdorfer, W.H. (1985) Towards a malaria vaccine - discussion. Parasitol. Today 1, 158-159

5 Hoffman, S.L. et al. (2002) Protection of humans against malaria by immunization with radiation-attenuated Plasmodium falciparum sporozoites. J. Infect. Dis. 185, 1155-1164

6 Long, C.A. and Hoffman, S.L. (2002) Parasitology: malaria - from infants to genomics to vaccines. Science 297, 345-347

7 Moorthy, V.S. et al. (2004) Malaria vaccine developments. Lancet 363, 150-156

8 Kaslow, D.C. (2004) A potential disruptive technology in vaccine development: gene-based vaccines and their application to infectious diseases. Trans. R. Soc. Trop. Med. Hyg. 98, 593-601

9 Ballou, W.R. et al. (2004) Update on the clinical development of candidate malaria vaccines. Am. J. Trop. Med. Hyg. 71 (Suppl. 2), 239-247

10 Bojang, K.A. et al. (2001) Efficacy of RTS,S/AS02 malaria vaccine against Plasmodium falciparum infection in semi-immune adult men in The Gambia: a randomized trial. Lancet 358, 1927-1934

11 Alonso, P.L. et al. (2004) Efficacy of the RTS,S/AS02A vaccine against Plasmodium falciparum infection and disease in young African children: randomized control trial. Lancet 364, 1411-1420

12 Smith, P.G. and Milligan, P.J. (2004) Malaria vaccine: 3 or 6 months' protection? Lancet $365,472-473$

13 Tsuji, M. and Zavala, F. (2003) T cells as mediators of protective immunity against liver stages of Plasmodium. Trends Parasitol. 19, 88-93

14 Sedegah, M. et al. (1998) Boosting with recombinant vaccinia increases immunogenicity and protective efficacy of malaria DNA vaccine. Proc. Natl. Acad. Sci. U. S. A. 95, 7648-7653

15 Dunachie, S.J. and Hill, A.V.S. (2003) Prime-boost strategies for malaria vaccine development. J. Exp. Biol. 206, 3771-3779

16 Bejon, P. et al. (2005) Calculation of liver-to-blood inocula, parasite growth rates, and preerythrocytic vaccine efficacy, from serial quantitative polymerase chain reaction studies of volunteers challenged with malaria sporozoites. J. Infect. Dis. 191, 619-626

17 Moorthy, V.S. et al. (2004) A randomized, double-blind, controlled vaccine efficacy trial of DNA/MVA ME-TRAP against malaria infection in Gambian adults. PLoS 1, 1-16

18 Luke, T.C. and Hoffman, S.L. (2003) Rationale and plans for developing a non-replicating, metabolically active, radiation-attenuated Plasmodium falciparum sporozoite vaccine. J. Exp. Biol. 206, 3803-3808 
19 Plebanski, M. et al. (2005) Direct processing and presentation of antigen from malaria sporozoites by professional antigen-presenting cells in the induction of $\mathrm{CD} 8^{+}$T-cell responses. Immunol. Cell Biol. 83, 307-312

20 Tongren, J.E. et al. (2004) Malaria vaccines: if at first you don't succeed.... Trends Parasitol. 20, 604-610

21 Mueller, A.K. et al. (2005) Genetically modified Plasmodium parasite, as a protective experimental malaria vaccine. Nature 433, 164-167

22 Waters, A.P. et al. (2005) Malaria vaccines: back to the future? Science 307, 528-530

23 Gardner, M.J. et al. (2002) Nature. Genome sequence of the human malaria parasite Plasmodium falciparum 419, 498-511

24 Aguiar, J.C. et al. (2004) High throughput generation of Plasmodium falciparum functional molecules by recombinational cloning. Genome Res. 14, 2076-2082

25 Good, M.F. (2005) Vaccine-induced immunity to malaria parasites and the need for novel strategies. Trends Parasitol. 21, 29-34

26 Bull, P.C. et al. (1998) Parasite antigens on the infected red cell surface are targets for naturally acquired immunity to malaria. Nat. Med. 4, 358-360

27 Genton, B. et al. (2002) A recombinant blood-stage malaria vaccine reduces Plasmodium falciparum density and exerts selective pressure on parasite populations in a Phase 1-2b trial in Papua New Guinea. J. Infect. Dis. 185, 820-827

28 Crewther, P.E. et al. (1996) Protective immune responses to apical membrane antigen 1 of Plasmodium chabaudi involve recognition of strain-specific epitopes. Infect. Immun. 64, 3310-3317

29 Martinelli, A. et al. (2005) A genetic approach to the de novo identification of targets of strain-specific immunity in malaria parasites. Proc. Natl. Acad. Sci. U. S. A. 102, 814-819

30 Gruner, A.C. et al. (2003) Pre-erythrocytic antigens of Plasmodium faciparum: from rags to riches. Trends Parasitol. 19, 74-78

31 Oeuvray, C. et al. (1994) Merozoite surface protein-3: a malaria protein inducing antibodies that promote Plasmodium falciparum killing by cooperation with blood monocytes. Blood 84, 1594-1602

32 Bull, P.C. et al. (2000) Plasmodium falciparum-infected erythrocytes: agglutination by diverse Kenyan plasma is associated with severe disease and young host age. J. Infect. Dis. 182, 252-259

33 Stanisic, D.I. et al. (2003) Analysis of immunological non-responsiveness to the 19-kilodalton fragment of merozoite surface protein 1 of Plasmodium yoelii: rescue by chemical conjugation to diphtheria toxoid (DT) and enhancement of immunogenicity by prior DT vaccination. Infect. Immun. 71, 5700-5713

34 Urban, B.C. et al. (1999) Plasmodium falciparum-infected erythrocytes modulate the maturation of dendritic cells. Nature 400, 73-77
35 Good, M.F. et al. (2004) The immunological challenge to developing a vaccine to the blood stages of malaria parasites. Immunol. Rev. 20l, 254-267

36 Pombo, D.J. et al. (2002) Immunity to malaria after administration of ultra-low doses of red cells infected with Plasmodium falciparum. Lancet 360, 610-617

37 Stevenson, M.M. and Riley, E.M. (2004) Innate immunity to malaria. Nat. Rev. Immunol. 4, 169-180

38 Makobongo, M.O. et al. (2003) The purine salvage enzyme hypoxanthine guanine xanthine phosphoribosyl transferase is a major target antigen for cell-mediated immunity to malaria. Proc. Natl. Acad. Sci. U. S. A. 100, 2628-2633

39 Staalsoe, T. et al. (2004) Variant surface antigen-specific IgG and protection against clinical consequences of pregnancy-associated Plasmodium falciparum malaria. Lancet 363, 283-289

40 Tuikue Ndam, N.G. et al. (2005) High level of var2csa transcription by Plasmodium falciparum isolated from the placenta. J. Infect. Dis. 192, 331-335

41 Jensen, A.T.R. et al. (2004) Plasmodium falciparum associated with severe childhood malaria preferentially expresses PfEMP1 encoded by group A var genes. J. Exp. Med. 199, 1179-1190

42 Carter, R. (2001) Transmission blocking malaria vaccines. Vaccine 19, 2309-2314

43 Kaslow, D.C. (2002) Transmission-blocking vaccines. Chem. Immunol. 80, 287-307

44 Coban, C. et al. (2004) Induction of Plasmodium falciparum transmission-blocking antibodies in nonhuman primates by a combination of DNA and protein immunizations. Infect. Immun. 72, 253-259

45 Malkin, E.M. et al. (2005) Phase 1 vaccine trial of PvS25H: a transmission blocking vaccine for Plasmodium vivax malaria. Vaccine 23 , 3131-3138

46 Khan, S.M. et al. (2005) Proteome analysis of separated male and female gametocytes reveals novel sex-specific Plasmodium biology. Cell 121, 675-687

47 Struik, S.S. and Riley, E.M. (2004) Does malaria suffer from lack of memory? Immunol. Rev. 201, 268-290

48 Greenwood, B.M. et al. (2005) Malaria. Lancet 365, 1487-1498

49 Walther, M. et al. (2004) Safety, immunogenicity and efficacy of a preerythrocytic malaria candidate vaccine, ICC-1132 formulated in Seppic ISA 720. PloS Med 1, e33

50 Lopez, J.A. et al. (2001) A synthetic malaria vaccine elicits a potent $\mathrm{CD}^{+}$and $\mathrm{CD}^{+} \mathrm{T}$ lymphocyte immune response in humans. Implications for vaccine strategies. Eur. J. Immunol. 31, 1989-1998

51 Miller, L.H. et al. (1986) Research towards malaria vaccines. Science $234,1349-1356$

\section{Reproduction of material from Elsevier articles}

Interested in reproducing part or all of an article published by Elsevier, or one of our article figures? If so, please contact our Global Rights Department with details of how and where the requested material will be used. To submit a permission request on-line, please visit:

http://www.elsevier.com/wps/find/obtainpermissionform.cws_home/obtainpermissionform

Alternatively, please contact:

Elsevier

Global Rights Department

PO Box 800,

Oxford OX5 1DX, UK.

Phone: $(+44)$ 1865-843830

Fax: (+44) 1865-853333

permissions@elsevier.com 